\title{
Educación en Nutrición Clínica
}

\section{Clinical Nutrition Education}

\author{
Dr. Humberto Arenas Márquez
}

https://doi.org/10.35454/rncm.v1n2.039

Aprender es cambiar. La educación es un proceso que cambia al alumno.

George Leonard.

\section{¿POR QUÉ ES NECESARIO MEJORAR LA EDUCACIÓN EN NUTRICIÓN CLÍNICA?}

Evidencias demuestran que la tasa de prevalencia de malnutrición es alarmante y que los costos asociados a ella se incrementan en forma exponencial, pero por otro lado la óptima nutrición está íntimamente relacionada con el resultado final positivo del manejo de una enfermedad; por tanto, idóneamente, un plan nutricional debería aplicarse a todo enfermo que ingrese a un hospital; sin embargo, la realidad es distinta; el médico líder del equipo de atención no ha sido entrenado para afrontar este reto y lo que es peor, no está consciente de su necesidad ${ }^{(1-3)}$.

La mala práctica de la nutrición clínica es producto de una enseñanza inadecuada.

Por tanto, el reto actual de los expertos en Nutrición Clínica es desarrollar herramientas para el aprendizaje y concientización de la importancia de la terapia nutricional entre los profesionales de la salud, en especial médicos y nutricionistas así como fomentar el desarrollo profesional interdisciplinario en la atención nutricional ${ }^{(4)}$.

\section{¿CUÁNDO Y A QUIÉN EDUCAR?}

Idóneamente todas las profesiones relacionadas con la salud deberían aprender al menos conceptos básicos de nutrición clínica, de tal forma que pudiesen mejorar su autoconsciencia de la necesidad de nutrir a su paciente en forma óptima en todos los tiempos y bajo todas las circunstancias. Pero cuando no se tiene el conocimiento para proporcionar una terapia nutricional óptima, reco-

1 Líder Unidad de Práctica Integrada en Falla Intestinal. Hospital San Javier. Guadalajara, México. Presidente de la Felanpe 2016-2018. nocer el no saber y solicitar ayuda al profesional experto es de vital importancia.

El reconocimiento de la nutrición clínica como una disciplina autónoma es un nuevo fenómeno que llevará inevitablemente a algunos ajustes de los límites profesionales existentes ${ }^{(5)}$.

Una especialidad interdisciplinaria debe ser reconocida y esta debe ser una verdadera especialidad. Más investigación y esfuerzos deben ser combinados para determinar las competencias y contenidos de la nutrición clínica.

También resulta fundamental concientizar y educar al paciente acerca los riesgos del ayuno prolongado y de los beneficios de la Nutrición Clínica, así como de su derecho a participar en toma de decisiones y colaborar en acciones que puedan repercutir en su propia seguridad.

\section{¿CÓMO EDUCAR?}

La teoría del aprendizaje del adulto sugiere que las habilidades, los conocimientos, las actitudes y los hábitos deben ser adquiridos a través de contextos auténticos y comunicándose con sus compañeros y expertos dentro $y$ sobre esos contextos ${ }^{(6)}$.

El aprendizaje del adulto condena la inefectividad de la educación médica continua la cual es manejada más por el maestro que por el aprendiz. Por otro lado, existen pocos médicos especializados en nutrición clínica. Además la educación tradicional es didáctica más que interactiva y finalmente se encuentra concentrada en unos cuantos más que distribuida.

El nuevo paradigma es el desarrollo profesional continuo, siguiendo los principios de aprendizaje del adulto: basado en autodirección, experiencias, interés por aprender, orientación en el aprendizaje y motivación de aprendizaje con el objetivo de modificar el patrón de práctica ${ }^{(7,8)}$.

Sin duda las competencias duras ligadas al conocimiento y habilidades en nutrición clínica deben ser desarrolladas; sin embargo, no menos importantes son las competencias blandas, aquellas relacionadas con la 
integración del comportamiento de la persona, su desempeño social, liderazgo y manejo emocional. Por otra parte dichas competencias están asociadas a la personalidad, las emociones y a valores adquiridos basadas en la inteligencia emocional, ya que es solo en el balance entre las competencias técnicas y las no técnicas cuando se puede lograr la efectividad profesional.

Es importante en el proceso de aprendizaje de la Nutrición Clínica modificar conductas y demostrar el valor de la atención nutricional a través de una detección temprana, diagnóstico correcto, implementación de la terapia y monitoreo apropiado, lo cual sin duda repercutirá en el resultado y en el costo de atención ${ }^{(9)}$. Pero también se debe crear autoconciencia demostrando las consecuencias de una atención nutricional deficiente tanto para el paciente como para el sistema en el cual trabajamos y la forma como podemos rescatar nutricionalmente a estos pacientes.

La clave para el aprendizaje en los tiempos modernos es la interacción; por tanto, el papel del educador es ser un buen facilitador del aprendizaje a través de la pregunta correcta con la retroalimentación inmediata.

No es solo el conocimiento, los protocolos, las guías de manejo y las evidencias las que van a mejorar el proceso de la educación en la atención nutricional. Es fundamental también desarrollar la actitud positiva del proveedor de la atención nutricional y la cultura del trabajo en equipo de alto desempeño como características esenciales para proveer la óptima terapia nutricional.

\section{QUIÉN}

Cambiar la cultura siempre es lo más difícil, pero es una urgencia. Más de $70 \%$ de los maestros hoy en día utilizan el modelo tradicional de educación a través de conferencias y este es el status quo de la educación desde hace siglos.

Nuestra meta como educadores debería ser la transformación de un novato a un profesional competente, a un experto y finalmente a un maestro. Por décadas, la investigación ha confirmado, que el gran factor por el que los estudiantes aprenden, es por la calidad de los maestros. Lo memorable siempre será la profunda huella interpersonal del maestro.

\section{DÓNDE}

Se ha mencionado por siglos que la clínica se aprende al lado del enfermo. En el pase de visita diaria en cualquier servicio debería esperarse que en el análisis del caso siempre surgiera el tema de la nutrición óptima para cada paciente en particular ${ }^{(10)}$.

La cultura nutricional es demostrada a través de los hábitos que tenemos, dicha cultura es adquirida en el ambiente donde nos desarrollamos y practicamos. Por tanto la cultura nutricional es todo lo que has aprendido, lo que haces y como lo haces.

Para enfrentar el déficit en la atención nutricional que proporcionamos a nuestros pacientes, deberíamos utilizar tres competencias innatas e individuales del liderazgo: la inteligencia, el carisma y el esfuerzo. Sin embargo, rara vez las utilizamos en conjunto. Estas capacidades permiten ayudar a otros y aprender de nuestras experiencias de cada día. Nosotros, los líderes de la Nutrición Clínica deberíamos inspirar a nuestros colaboradores a actuar en forma similar para así ser capaces de crear y desarrollar nuestra esfera de influencia. Esta es la ruta que se debe utilizar para crear una cultura nutricional en los hospitales donde laboramos.

Resulta pues fundamental desarrollar en el ambiente hospitalario la cultura de seguridad en la atención nutricional basada en el compromiso, responsabilidad, participación, comunicación y confianza, utilizando la retroalimentación para construir el aprendizaje del equipo en la atención nutricional ${ }^{(11)}$.

También es fundamental utilizar la tecnología de la información para acceder al mayor número de profesionales de la salud.

El aprendizaje en línea es aprender con las fuentes disponibles en la Internet, incluyendo libros, videos, lecciones y software.

Las tres características del aprendizaje en línea son:

1. Retro-alimentación inmediata

2. El estudiante es dueño del aprendizaje

3. El profesor no desempeña el papel central.

Los docentes que enseñen en línea deben estar atentos a mantener a sus estudiantes comprometidos. También necesitan ser capaces de seleccionar contenidos atractivos, recursos multimedia ricos para la instrucción, métodos de instrucción de entrega de contenidos no tradicionales; una sólida filosofía de enseñanza; saber usar el Internet para aprender y enseñar estrategias de instrucción innovadoras.

\section{HABILIDADES DEL SIGLO XXI}

Más allá del conocimiento de dominio específico de la nutrición existen otras habilidades consideradas no 
cognitivas y que son importantes en el siglo XXI, estas podrían ser mejor descritas como características individuales, las cuales no se miden ni cuantifican con facilidad mediante las pruebas tradicionales. Implican actitudes personales y habilidades sociales. ¿Cuáles son?: aquellas que promueven el pensamiento innovador y se conocen como las $4 \mathrm{C}$ :

1. Capacidad crítica y autocrítica. Los individuos que aceptan y hacen la crítica de lo que hacemos y leemos son aquellos cuya inteligencia se desarrolla y cada oportunidad los lleva a un aprendizaje.

2. Comunicación. Es una de las mejores medicinas, milenaria por cierto y la base de las relaciones humanas. Para que sea exitosa se requiere una buena comunicación bidireccional. Desafortunadamente, la comunicación no ha sido contemplada en el periodo de la formación académica y universitaria. Es necesario que los profesionales aumenten la capacidad de utilizar la comunicación en forma eficaz.

3. Colaboración. Solamente trabajando juntos como equipo mejoraremos la calidad y la seguridad del paciente en diversos escenarios de atención nutricional que van desde el hospital hasta su domicilio. El contar con una plataforma multidisciplinaria nos permite una evaluación y re-evaluación constante de las condiciones nutricionales del paciente, construyendo planes de atención y metas terapéuticas a corto, mediano y largo plazo. Esto requerirá un modelo interdependiente donde la comunicación constante permita el máximo desempeño del equipo, comprometiéndose todos y cada uno de ellos en el logro de las metas del paciente, pero con la mayor seguridad ${ }^{(12)}$.

4. Creatividad e Innovación. La creatividad es un hábito, y la mejor creatividad es el resultado de hábitos que trabajan bien. La innovación por otro lado es la habilidad de algunos para cambiar ideas viejas por ideas nuevas, para modificar principios ya establecidos y para retar lo ordinario.

\section{FACTORES SOCIALES Y EMOCIONALES EN EL APRENDIZAJE}

El aprendizaje social y emocional se ha implementado como estrategia para dotar a los estudiantes de las habilidades emocionales y psicológicas que afectan de manera fundamental sus logros tanto dentro como fuera del aula (inteligencia emocional), como son: la autoregulación, la autoconsciencia, la toma responsable de decisiones, las habilidades en relaciones y la consciencia social. Los programas con estos contenidos, bien diseñados y bien implementados, generan resultados académicos, sociales, emocionales y conductuales positivos.

Las habilidades para mantener la calma bajo presión y relacionarse bien con los demás son cruciales para la comunicación productiva y la colaboración. Además, la creatividad implica tomar riesgos, superar obstáculos y retrasar la gratificación. Además, para lograr un pensamiento crítico, hay que llevar a cabo procesos de análisis y resolución de problemas, lo cual puede ser un reto con resultados impredecibles.

La meta de un plan de estudios en nutrición clínica es ayudar a los estudiantes a "aprender a saber nutrición clínica", "aprender a hacer nutrición clínica" y "aprender a ser una diferencia en la atención nutricional" (13-17).

\section{EL EFECTO DE LA EVALUACIÓN}

Las evaluaciones a través de casos clínicos son formativas. El presentar un caso problema y hacer preguntas inteligentes con base en una acción, lleva al alumno a tomar decisiones. El resultado de dicha evaluación es fundamental para el alumno y el maestro, ya que ambos deben reconocer las competencias que deben reforzarse y mejorarse.

En cada evaluación se sugiere incluir evaluaciones de competencias duras y blandas y ante cada respuesta proporcionar retroalimentación inmediata basada en evidencia y también en el sentido común con el fin de modificar pensamientos o conductas que mejoren el aprendizaje.

Aprender del error en la práctica de la nutrición clínica, es esencial.

Se recomienda responder tres preguntas fundamentales: ¿por qué pasó?, ¿cómo resolverlo? y ¿cómo se podría haber prevenido?

En cada caso adopta el hábito de la reflexión: ¿qué harías diferente la próxima vez?

\section{REFLEXIONES FINALES}

Son pues necesarias hacer reformas en la educación de la nutrición clínica de los profesionales de la salud involucrados en la atención nutricional de enfermos, que promuevan el desarrollo de habilidades del pensamiento así como modificaciones de conductas y actitudes que permitan una mejora en la calidad y seguridad de la atención nutricional ${ }^{(18,19)}$.

La reforma en la educación se basa en una transformación hacia una "economía del conocimiento de la nutrición” impulsada por la tecnología de la información, 
incluyendo un conjunto de metas como el cultivo de la innovación, la formación del carácter y la búsqueda de un resultado final hacia una educación en nutrición orientada a la calidad en el servicio de la atención nutricional ${ }^{(20)}$.

Hoy, tanto maestros como alumnos tenemos que pensar, tomar riesgos, y no simplemente seguir instrucciones. Tenemos las herramientas y las habilidades necesarias para cambiar el aula y convertir el aprendizaje de la Nutrición Clínica en un proceso emocionante y relevante para estudiantes, maestros y en especial para los pacientes y la sociedad a la cual debemos defender ${ }^{(21)}$.

\section{Referencias bibliográficas}

1. Waitzberg D, Caiaffa W, Correia I. Hospital Malnutrition: The Brazilian National Survey (IBRANUTRI): A Study of 4000 Patients. Nutrition. 2001; 17:573-80.

2. Correia I, Campos J. Prevalence of hospital Malnutrition in Latin America: The Multicenter ELAN study. Nutrition. 2003;19:823-5.

3. Abbassi J. Stephen Devries, MD: Training physicians about Nutrition. JAMA. 2018;319:1751-1752.

4. Correia I, Hegazi R, Diaz J. Addressing Disease-Related Malnutrition in Healthcare: A Latin American Perspective. JPEN J Parenter Enteral Nutr. 2016; 40 (3):319-25.

5. Cardenas D. What is clinical nutrition? Understanding the epistemological foundations of a new discipline. Clinical Nutrition. 2016(11): e63 - e66.

6. Santibañes E, Cano V, Pellegrini C. Excellence in surgery: Becoming the "best" you can be. ACS Bulletin April 2018:10-6.

7. Arenas $\mathrm{H}$. El futuro de la seguridad del paciente y la calidad en la atención médica. En: Perez JA. Seguridad del paciente al alcance de todos. Editorial Alfil: AMCG; 2013. p. 495-514.

8. Kris-Etherton P, Akabas S, Bales C, Bistrian B, et al. The need to advance nutrition education in the training of health care professionals and recommended research to evaluate implementation and effectiveness. Am J Clin Nutr. 2014;99:1153S-66S.

9. Tappenden K, Quatrara B. Critical Role of Nutrition in Improving Quality of Care. An Interdisciplinary Call to Action to Address Adult Hospital Malnutrition. JPEN J Parenteral Enteral Nutr. 2013; 37:482-97.
10. Castro M, Pompilio C, Horie L, Gimenez C, Waitzberg D. Education program on medical nutrition and length of stay of critically ill patients. Clinical Nutrition. 2013;(32):1061-6.

11. Frenk J, Chen L, Bhutta Z, et al. Health professionals for a new century: transforming education to strengthen health systems in an interdependent world. The Lancet. 2010; (376):1923 -58.

12. Arenas H, García J, López M. Medidas de prevención en el paciente quirúrgico; En AMCG. Tratado de Cirugía General 3a Edición. CDMX: Manual moderno. 2017.p. 208-17.

13. Kris-Etherton P, Akabas S, Douglas P, Kohlmeier M, et al. Nutrition Competencies in Health Professionals' Education and Training: A New Paradigm. American Society for Nutrition. Adv. Nutr. 2015;6:83-7. doi:10.3945/ an.114.006734.

14. Daley B, Cherry-Bukowiec J, Van Way III C, Collier B, et al. Current Status of Nutrition Training in Graduate Medical Education from a Survey of Residency Program Directors: A Formal Nutrition Education Course Is Necessary. JPEN J Parenter Enteral Nutr. 2016; 40:95-9.

15. Dunlosky J, Rawson K, Marsh E, Nathan M, Willingham D. Improving Students Learning with Effective Learning Techniques Promising Directions From Cognitive and Educational Psychology. Psychological Science. 2013;14(1):4-58.

16. Kiraly L, McClave S, Neel D, Evans D, et al. Physician Nutrition Education. Nutr Clin Pract. 2014;29:332-7.

17. Brill J, August D, DeLegge M, Hegazi R, et al. A Vision of the Future for Physician Practice in Nutrition. JPEN J Parenter Enteral Nutr. 2010; 34:86S-96S.

18. Karim S, Ibrahim B, Tangiisuran B, Davies G, et al. What Do Healthcare Providers Know About Nutrition Support? A Survey of the Knowledge, Attitudes, and Practice of Pharmacists and Doctors Toward Nutrition Support in Malaysia. JPEN J Parenter Enteral Nutr. 2015; 39:482-9.

19. Arenas $\mathrm{H}$. Calidad en salud y su relación con el proceso de certificación y recertificación. Cirujano General. 2002;(1):72-5.

20. Arenas $\mathrm{H}$. La reforma en la enseñanza y práctica de la cirugía general. Cir Gen. 1996; 18: 53-8.

21. Arenas D, Plascencia A, Ornelas D, Arenas H. Hospital Malnutrition Related to Fasting and Underfeeding Is It an Ethical Issue? NCP. 2016;31(3):316-24. 